\title{
Correlation of Placental Morphology and Umblical Doppler Flow Velocimetry with Fetal Outcome
}

\author{
A.I.El-Mashad, M.A.Mahmoud, A.E.Mansour, T.M.Assar and M.H.Abdel Rahman \\ Obstetrics,Gynecology Dept., Faculty of Medicine, Benha Univ., Benha, Egypt \\ E-Mail:Marwa@gmail.com
}

\begin{abstract}
This examination was assessed the connection between ultrasonographic placental attributes (localisation ,thickness) and fetal umblical doppler stream velocimetry with fetal outcome.This study led at Department of Obstetrics and Gynecology Benha University Hospital, where the patients going to obstetrics outpatient Clinic. During the investigation time frame from June 2017 to May 2018. This investigation included 66 pregnant ladies whose gestational age at 24 weeks incubation from the last typical feminine time frame affirmed by early ultrasound assessment during the first trimester were found in the antenatal center and were followed up at 32 and 36 weeks. Point by point history was taken to preclude clinical and careful ailments that could influence the investigation. US assessment fetal biometry Then assessment of fetal weight (EFW) determined by the recipe of Hadlock et al.,placental thickness ( The placental thickness was estimated by setting the ultrasound transducer oppositely to the plane of the placenta, in the region of the line inclusion, close to the mid-placental bit) and umbilical Doppler examination.All were performed at three visits : first somewhere in the range of 24 and 28weeks, second between 28and 32 weeks, and third somewhere in the range of 32 and 36weeks and fetal result .The investigation indicated measurably critical increment placental thickness in the principal, second and third visit were 25.39 $\pm 1.88,30.62 \pm 3.25,36.42 \pm 4.3$ and fetal load in the main, second and third visit were $24.26 \pm 1.11,30.17 \pm 1.83,33.55 \pm$ 2.14.There is non-huge connection between placental breadth and umbilical conduit stream in the primary second and third visits. There is measurably non-critical connection between's placental breadth in the third visit and APGAR score .So estimations of placental Thickness ought to be incorporated as discrete things on routine U/S reports and can be remembered for sonographic equation with different boundaries as BPD, FL and AC for most exact fetal weight assessment.
\end{abstract}

Keywords: Placental Thickness, Second and Third Trimester, Ultrasonography, Umblical doppler .

\section{Introduction}

An ordinary pregnancy is a review term for the great state of the embryo at term somewhere in the range of 37 and 42 weeks. The measures of an ordinary pregnancy are conveyance of a solitary solid child, with fetal load of ( 2.5 - 4) $\mathrm{kg}$ and with no maternal complexity [20] .

Placenta is an intrauterine combination of fetal and maternal tissues with the end goal of physiological exchange of supplements and oxygen from mother to baby and move of side-effects of digestion from hatchling to mother for continuation of fetal life. The intrauterine presence of embryo is subject to this fundamental organ and the placenta has been considered as a significant pointer for fetal and maternal infections [20].

Placental thickness is a lot of identified with fetal turn of events and might be a key in perinatal result. At term the placenta is roughly $3 \mathrm{~cm}$ thick and measures 15 $25 \mathrm{~cm}$ in width [2].

A'warning limit' of placental width of $8 \mathrm{~cm}$ and placental thickness of $2 \mathrm{~cm}$ at 36 weeks predicts low birth weight children .Small placentas are related with toxemia, chromosomal anomalies, serious maternal diabetes mellitus, constant fetal contaminations and intrauterine development limitation . The placentas more than $4 \mathrm{~cm}$ thick at term have been seen in conditions like diabetes mellitus, perinatal diseases, hydrops fetalis (both resistant and non insusceptible) [19].

Placental blood flow and gas and supplement transport are significant determinants of fetal development [17]. The size, weight and state of the placenta are generally dependent upon wide varieties and placental size is identified with its capacity to move supplements. A few investigations have portrayed the connection between placental morphometry and antagonistic pregnancy result, including fetal development limitation (FGR) [21].

Placental capacity can be evaluated in vivo by uteroplacental Doppler flow velocimetry and fetal development can be surveyed by sequential ultrasonic biometry. Before pregnancy, flow speed wave structures in the uterine course (UtA) will in general be high resistance, and the advancement of a low opposition example of flow in the first half of pregnancy is believed to be because of attack of the maternal obstruction vessels by the trophoblast [18] .

Diligence of high opposition examples of flow in the UtA in mid-development has been related with an expanded danger of obstetric entanglements. The flow speed wave structure in the umbilical supply route (UmA) is typically low opposition in the last trimester of pregnancy,and this is thought to reflect the advancement of the villous vascular tree [21] .

A high opposition example of flow in the umbilical course is broadly utilized as a marker of placental brokenness, where the underlying connect of high obstruction flow is maldevelopment of the tertiary villi [17].

Most investigations on the between connections between antenatal utero-placental Doppler blood flow velocimetry and the post-natal findings have zeroed in on the minuscule and ultrastructural attributes of the 
placenta and placental bed. In any case, uteroplacental blood flow could likewise be identified with the gross morphology of the placenta [21] .

\section{Patients and methods}

This prospective study has been conducted at Department of Obstetrics \& Gynecology Benha University Hospital,where the patients attending obstetrics outpatient Clinic, after appraise local ethical committee,consent obtained from the patients who were included in the study after explaining to them their condition.

\subsection{The women who were included in our study has} the following inclusion criteria

Known last menstrual period ,Singleton pregnancy,Age group of 20 - 35 year and normal BMI between $\left(25 \_29.9 \mathrm{~kg} / \mathrm{m} 2\right)$.

\subsection{While women who were exclused has the following criteria}

Patients who are not sure of dates or with history of irregular cycles,Previous CS ,Chronic medical diseases like diabetes, hypertension, chronic renal disease, Obese females (BMI 30 or higher), Multiple pregnancy , Congenital anomaly in fetus, Low lying placenta

66 pregnant ladies who fullfill the inclusion criteria were subjected to the following at the first visit : History taking in details about general health and previous

pregnancy and delivery,Physical examination to exclude any disease and obestatric examination. Then at 28,32,36 week of gestation all patient will be subjected to

\subsection{Ultrasound Technique of ultrasound examination}

The fetus was observed for viability and gross anatomical defects and gestational age was estimated using various growth parameters:Biparietal Diameter (BD), Femur Length (FL), Abdominal Circumference (AC), Head Circumference (HC) and estimated fetal weight (EFW). Placental thickness was taken at 24, 32 and 36 weeks. The placental thickness was measured at the level of umbilical cord insertion in longitudinal direction from the lateral chorionic plate to the cord insertion.Umbilical artery color Doppler was used for further reconfirmation of the site of umbilical cord insertion.

\subsection{Doppler studies}

Pulsed wave high-resolution color Doppler ultrasonography (Voluson S6 KT-LM190SDG1V 2014) Was used to identify and to obtain blood flow velocity waveforms from the Umbilical artery, from a free loop of the umbilical cord.umblical artery flow has characteristic saw tooth appearance of arterial flow in one direction and continuous umbilical venous blood flow in the other. fetal outcome with normal healthy baby

\section{Results}

Table (1) Distribution of the studied participants according to demographic characteristics and obstetric history.

\begin{tabular}{lc}
\hline Parameters & $\mathbf{N}=\mathbf{6 6}$ \\
\hline Age (years) & \\
Mean \pm SD & $28.06 \pm 5.2$ \\
Range & $20-35$ \\
Gravidity & \\
Mean \pm SD & $3.27 \pm 1.02$ \\
Median (range) & $3(1-3)$ \\
Parity & \\
Mean \pm SD & $1.47 \pm 0.66$ \\
Median (range) & $1(1-3)$ \\
Abortion & \\
Mean \pm SD & $0.7 \pm 0.66$ \\
Median (range) & $1(0-2)$ \\
\hline
\end{tabular}

Age of the studied participants ranged from 20 to 35 years with mean 28.06 years. Gravidity ranged from 1 to
3 with median 3 . Parity ranged from 1 to 3 with median 1 while previous abortions ranged from 0 to 2 with median 1 .

Table (2) Change in placental diameter over time.

\begin{tabular}{lccccc}
\hline Placental diameter & Mean \pm SD & Range & t & P & \% change from 1 $^{\text {st }}$ visit \\
First visit (24-28 weeks) & $25.39 \pm 1.88$ & $22-28$ & & & $\begin{array}{c}\text { Median (range) } \\
\text { Second visit (28-32 weeks) }\end{array}$ \\
Third visit (32-36 weeks) & $30.62 \pm 3.25$ & $26-36$ & -19.516 & $<0.001 * *$ & $4.41-40)$ \\
P (F) & $36.42 \pm 4.3$ & $30-41$ & -17.323 & $<0.001 * *$ & $45.83(15.38-60)$ \\
\hline
\end{tabular}

$\mathrm{T}$ paired sample $\mathrm{t}$ test $\mathrm{f}$ repeated measure anova $* * \mathrm{p}<0.001$ is statistically highly significant.there is statistically significant increase in placental diameter over time. On comparing each two successive visits, the difference is significant. 
Table (3) Change in Biparietal diameter over time.

\begin{tabular}{lllll}
\hline BPD & Mean \pm SD & Range & T & p \\
\hline First visit (24-28 weeks) & $25.67 \pm 2.09$ & $22-28$ & & $<0.001^{* *}$ \\
Second visit (28-32 weeks) & $30.15 \pm 2.62$ & $26-36$ & -27.964 & $<0.001^{* *}$ \\
Third visit (32-36 weeks) & $37.36 \pm 2.74$ & $30-41$ & -30.682 & \\
P (F) & \multicolumn{2}{c}{$<0.001^{* *}$} \\
\hline
\end{tabular}

T Paired Sample T Test F Repeated Measure Anova **P $<0.001$ Is Statistically Highly Significant

Table (4) Change in femur length over time.
There is statistically significant increase in (BPD) over time. On comparing each two successive visits, the difference is significant.

\begin{tabular}{lcccc}
\hline FL & Mean \pm SD & Range & T & P \\
\hline First visit (24-28 weeks) & $25.39 \pm 1.94$ & $22-28$ & & $<0.001 * *$ \\
Second visit (28-32weeks) & $29.64 \pm 2.2$ & $26-33$ & -20.047 & $<0.001^{* *}$ \\
Third visit (32-36 weeks) & $33.42 \pm 2.57$ & $30-36$ & -15.415 & \\
P (F) & \multicolumn{2}{c}{$<0.001 * *$} \\
\hline
\end{tabular}

$\mathrm{T}$ Paired sample $\mathrm{t}$ test $\mathrm{F}$ repeated measure ANOVA $* * \mathrm{p}<0.001$ is statistically highly significant.There is statistically significant increase in femur length over time. On comparing each two successive visits, the difference is significant

Table (5) Change in abdominal circumference over time.

\begin{tabular}{|c|c|c|c|c|}
\hline $\mathbf{A C}$ & Mean \pm SD & Range & $\mathbf{t}$ & $\mathbf{P}$ \\
\hline First visit (24-28 weeks) & $24.26 \pm 1.11$ & $22-26$ & & \\
\hline Second visit (28-32weeks) & $30.17 \pm 1.83$ & $28-33$ & -22.908 & $<0.001 * *$ \\
\hline Third visit (32-36 weeks) & $33.55 \pm 2.14$ & $30-36$ & -18.871 & $<0.001 * *$ \\
\hline $\mathbf{P}(\mathbf{F})$ & \multicolumn{3}{|c|}{$<0.001 * *$} & \\
\hline
\end{tabular}

$\mathrm{T}$ Paired sample $\mathrm{t}$ test $\mathrm{F}$ repeated measure ANOVA $* * p<0.001$ is statistically highly significant.There is

Table (6) Change in estimated fetal weight over time. statistically significant increase in abdominal circumference over time. On comparing each two successive visits, the difference is significant

\begin{tabular}{lcccc}
\hline EFW & Mean \pm SD & Median (Range) & T & P \\
\hline First visit (24-28 weeks) & $796.52 \pm 249.89$ & $420-1100$ & \\
Second visit (28-32weeks) & $1613.18 \pm 536.64$ & $700-2200$ & -19.703 & $<0.001^{* *}$ \\
Third visit (32-36 weeks) & $2434.85 \pm 491.07$ & $1500-3300$ & -15.069 & $<0.001^{* *}$ \\
P (F) & \multicolumn{2}{c}{$<0.001^{* *}$} & & \\
\hline
\end{tabular}

TPaired sample $t$ test $F$ repeated measure ANOVA $* * \mathrm{p}<0.001$ is statistically highly significant.There is statistically significant increase in estimated fetal weight over time. On comparing each two successive visits, the difference is significant.

Table (7) Change in umbilical artery flow by Doppler over time.

\begin{tabular}{lccccc}
\hline Umbilical artery Doppler & First visit & Second visit & Third visit & Q & p \\
\cline { 2 - 4 } & $\mathbf{N}=\mathbf{6 6}(\%)$ & $\mathbf{N}(\%)$ & $\mathbf{N}(\%)$ & & \\
\hline Abnormal & $6(9.1)$ & $4(6.1)$ & $2(3)$ & 6.0 & 0.05 \\
Normal & $60(90.9)$ & $42(93.9)$ & $64(97)$ & & \\
\hline
\end{tabular}

There is statistically non-significant change in umbilical artery Doppler over time. 


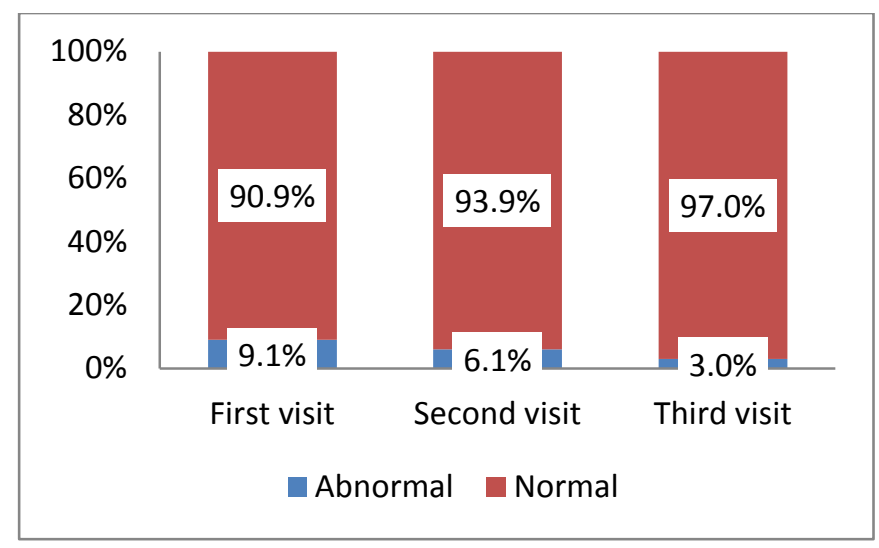

Fig (6) Compound bar chart showing umbilical artery colour Doppler among the studied participants.

Table (8) Relation between fetal color Doppler and placental diameter over time.

\begin{tabular}{|c|c|c|c|c|}
\hline & \multicolumn{2}{|c|}{ Placental diameter } & \multicolumn{2}{|c|}{ Test } \\
\hline & Mean \pm SD & Range & $\mathbf{t}$ & p \\
\hline The first vi & & & & \\
\hline Abnormal & $24.0 \pm 1.265$ & $22-27$ & -2.429 & 0.0 \\
\hline Normal & $25.53 \pm 1.88$ & $22-28$ & & $18 *$ \\
\hline The second & & & & \\
\hline Abnormal & $29.75 \pm 3.5$ & $26-35$ & -0.551 & 0.5 \\
\hline Normal & $30.68 \pm 3.25$ & $26-36$ & & 84 \\
\hline The third $v$ & & & & \\
\hline Abnormal & $33.33 \pm 5.77$ & $30-40$ & -1.279 & 0.2 \\
\hline Normal & $36.57 \pm 4.23$ & $30-41$ & & 05 \\
\hline
\end{tabular}

$\mathrm{T}$ Independent Sample $\mathrm{T}$ Test $\quad * \mathrm{P}<0.05$ Is $\quad$ the other hand, there is non-significant relation between Statistically Significant

There is significant relation between placental diameter and umbilical artery flow in the first visit. On placental diameter and umbilical artery flow in the second and third visits.

Table (9) Relation between fetus gender and placental diameter over time.

\begin{tabular}{|c|c|c|c|c|}
\hline \multirow{3}{*}{$\begin{array}{l}\text { Placental } \\
\text { diameter }\end{array}$} & \multicolumn{2}{|c|}{ Gender } & \multicolumn{2}{|c|}{ Test } \\
\hline & Male $(n=33)$ & Female $(n=33)$ & $\mathbf{t}$ & p \\
\hline & Mean \pm SD & Mean \pm SD & & \\
\hline First visit & $25.45 \pm 2.02$ & $25.33 \pm 1.76$ & -0.114 & 0.91 \\
\hline Second visit & $30.45 \pm 3.35$ & $30.79 \pm 3.19$ & -0.414 & 0.68 \\
\hline Third visit & $36.36 \pm 4.48$ & $36.48 \pm 4.19$ & 0.26 & 0.796 \\
\hline
\end{tabular}

t Independent sample t test.

There is non-significant relation between placental diameter fetus gender

Table (10) APGAR score of neonates.

\begin{tabular}{lc}
\hline APGAR & $\mathbf{N}=\mathbf{6 6}$ \\
\hline Mean \pm SD & $8.197 \pm 1.026$ \\
Range & $7-10$ \\
\hline
\end{tabular}

APGAR score of the studied patients ranged from 7 to 10 with mean 8.197. 
Table (11) Correlation between placental diameter and the studied parameters at the first visit.

\begin{tabular}{lcc}
\hline Parameters at the first visit & \multicolumn{2}{c}{ Placental diameter at the first visit } \\
\cline { 2 - 3 } & $\mathbf{r}$ & $\mathbf{p}$ \\
\hline EFW & 0.823 & $<0.001^{* *}$ \\
BPD & 0.76 & $<0.001^{* *}$ \\
FL & 0.824 & $<0.001^{* *}$ \\
AC & 0.37 & $0.002^{*}$ \\
Umbilical flow & 0.361 & $0.003^{*}$ \\
APGAR & 0.182 & 0.143 \\
\hline
\end{tabular}

$\mathrm{R}$ Pearson Correlation Coefficient $* \mathrm{P}<0.05$ Is Statistically Significant $* * \mathrm{P} \leq 0.001$ Is Statistically Highly Significant there is statistically significant positive correlation between placental diameter on the first visit and EFW, BPD, FL, AC and umbilical artery flow by Doppler on the first visit. There is statisticall.

y non-significant correlation between placental diameter in the third visit and APGAR score.

Table (12) Correlation between placental diameter and the studied parameters at the second visit.

\begin{tabular}{lcc}
\hline Parameters at the second & \multicolumn{2}{c}{ Placental diameter at the second visit } \\
\cline { 2 - 3 } visit & $\mathbf{r}$ & $\mathbf{p}$ \\
\hline EFW & 0.747 & $<0.001^{* *}$ \\
BPD & 0.784 & $<0.001^{* *}$ \\
FL & 0.718 & $<0.001^{* *}$ \\
AC & 0.682 & $<0.001^{* *}$ \\
Umbilical flow & 0.351 & $0.004^{*}$ \\
APGAR & 0.157 & 0.209 \\
\hline
\end{tabular}

$\mathrm{R}$ Pearson Correlation Coefficient $* \mathrm{P}<0.05$ Is Statistically Significant $* * \mathrm{P} \leq 0.001$ Is Statistically Highly Significant

there is statistically significant positive correlation between placental diameter on the second visit and EFW,
BPD, FL, AC and umbilical artery flow by Doppler on the second visit. There is statistically non-significant correlation between placental diameter in the third visit and APGAR score

Table (13) Correlation between placental diameter and the studied parameters at the third visit.

\begin{tabular}{lcc}
\hline Parameters at the third visit & \multicolumn{2}{c}{ Placental diameter at the third visit } \\
\cline { 2 - 3 } & r & P \\
\hline EFW & 0.728 & $<0.001^{* *}$ \\
BPD & 0.871 & $<0.001^{* *}$ \\
FL & 0.806 & $<0.001^{* *}$ \\
AC & 0.733 & $<0.001^{* *}$ \\
Umbilical flow & 0.253 & $0.04^{*}$ \\
APGAR & 0.158 & 0.204 \\
\hline
\end{tabular}

Pearson correlation coefficient $* p<0.05$ is statistically significant $* * \mathrm{p} \leq 0.001$ is statistically highly significant.there is statistically significant positive correlation between placental diameter on the first visit.and EFW, BPD, FL, AC and umbilical artery flow by Doppler on the first visit. There is statistically nonsignificant correlation between placental diameter in the third visit and APGAR score

\section{Discussion}

Typical placental capacities and construction is an important factor for the arrangement of a solid embryo and thus ordinary birth weight [17].
Satisfactory fetal development and resulting ordinary birth weight relies upon the effective conveyance of supplements from the mother to the baby by means of ordinarily working utero-placental organ. Typical advancement of placenta during development is vital for supporting of a solid hatchling [4].

In the principal trimester, placental development is more quick than that of the embryo. Yet, by roughly 17 postmenstrual weeks, placental and fetal loads are around equivalent. By term, placental weight is around one 6th of fetal weight. The normal placenta at term is $185 \mathrm{~mm}$ in breadth and $23 \mathrm{~mm}$ in thickness, with a volume of $497 \mathrm{ml}$ and a weight of $508 \mathrm{~g}$. These 
estimations change generally, and there are various variation placental structures and a few sorts of umbilical string inclusions [5].

Typical placental capacity and design is an important factor for the arrangement of a sound embryo and thus ordinary birth weight. Then again, any impedance in its improvement may profoundly affect fetal turn of events and pregnancy result [14]. S.Porat [14] suggested that sonographic addition of placental thick $\neg$ ness during second trimester is expected to overswelling of the intervillous space by maternal blood as opposed to by versatile development of utilitarian placental tissue.

A few parts of placental development including volume, weight, and plate territory were examined in various explores to discover their relationship with fetal anthropometry [4].

A few boundaries were utilized to survey fetal development to evaluate intrauterine ecological ampleness and fetal prosperity. Early location of any pathology in the placental bed and villi causes obstetrician to consider pre-birth care accurately [15].

The pace of placental development gives off an impression of being a significant determinant of birth weight, with the rate somewhere in the range of 17 and 20 weeks incubation being an indicator of fetal stomach and head perimeter, femoral length and biparietal distance across; more fragile affiliations are noticed for placental development before in pregnancy. A few parts of placental development including volume, weight, and plate territory were explored in various investigates to discover their relationship with fetal anthropometry [10].

The point of the investigation was to evalute the connection between placental morphology (localisation and thickness), fetal Doppler boundaries and fetal outcome.Age of the examined members went from 20 to 35 years with mean 28.06 years. Gravidity went from 1 to 3 with middle 3 . Equality went from 1 to 3 with middle 1 while past fetus removals went from 0 to 2 with middle 1.

Kartheek et al. [12] contemplated the placental histology and connect with shading stream doppler ultrasonography, maternal and neonatal result. Around 50 ladies chose for study were dispersed into control and high danger bunch concurring maternal qualities. Mean age of the contemplated members was 24.9 years.

In the current examination, there is genuinely critical expansion in placental distance across and BPD over the long run. On contrasting every two progressive visits, the thing that matters is critical. B.V.S.Kartheek [12] found that mean width of the placenta in benchmark group is $21.84 \mathrm{~cm}[9]$ evaluated the job of estimation of placental thickness and breadth in the third trimester utilizing twodimensional ultrasound in the assurance of low birth weight. They demonstrated a solid positive relationship between's neonatal weight and placental thickness, so placental thickness estimated at the degree of umbilical rope addition can be utilized as an exact sonographic pointer in the evaluation of fetal weight. Placental thickness underneath the tenth percentile was discovered to be related with low-birth-weight newborn children and poor Apgar score and expanded nursery confirmations.

In the current examination, there is measurably critical expansion in assessed fetal load after some time. On contrasting every two progressive visits, the thing that matters is huge. B.V.S.Kartheek [12] found that mean birth weight in benchmark group is $2.85 \mathrm{~kg}$.

In the current investigation, there is genuinely nonhuge change in umbilical vein Doppler over the long haul [19]. did an examination on 100 ladies with singleton pregnancy with high danger pregnancies, 21 pregnancies had irregular Doppler waveforms in umbilical corridors. Doppler waveform of the umbilical conduit was viewed as strange if S/D proportion and Resistance record (RI) surpasses 95th percentile or if there is missing or invert end diastolic stream in the embryos over the gestational age of 28 weeks.

Zhou et al. [16] explored whether the evaluation of placental perfusion utilizing 3D force Doppler ultrasonography could give a few insights for the expectation of macrosomia. They demonstrated that placental lists, for example, VI and FI, as gotten from 3D force Doppler ultrasonography, were essentially higher in third-trimester pregnancies that at last prompted babies experiencing macrosomia contrasted and typical controls. Be that as it may, neither one of the $3 \mathrm{ds}$ power Doppler lists, nor traditional 2D boundaries, end up being solid indicators for macrosomia.

In the current examination, there is huge connection between placental measurement and umbilical corridor stream in the three visits. N.Salavati [21] examined intraand interobserver reproducibility of 3-D-PDA investigation of put away datasets at different growths with a definitive objective being to build up a device for foreseeing placental brokenness. Ladies with a straightforward, reasonable singleton pregnancy were filtered at 12,16 or 20 weeks gestational age gatherings. The intraclass relationship coefficient (ICC) exhibited an elevated level of estimation unwavering quality at every incubation with intraobserver values .0 .90 and interobserver values of.0.6 for the vascular records. Flat Altman plots likewise indicated significant levels of arrangement. This investigation showed that 3-D-PDA information can be estimated dependably by various spectators from hid away as long as 18 weeks growth. Estimations become less solid as development propels with inclination between onlookers obvious at 20 weeks. [12] found that doppler stream was typical in all cases (100\%) in benchmark group.

In the current examination, there is genuinely huge positive connection between's placental width and EFW, BPD, FL, AC and umbilical vein stream by Doppler. U.Elchalal [6] found no connection was found between placental thickness and maternal age or equality. E.Aydin [2] decided the PT and the connection between the umbilical and uterine supply route Doppler boundaries and PT during the assessment of the fetal life structures during the subsequent trimester. We recommended that there was no connection between the placental thickness and obstetric Doppler boundaries. 
Abdelhamid et al. [1] assessed the relationship of placental thickness in the second and third trimesters with gestational age, weight, and neonatal result. They reasoned that estimation of placental thickness by ultrasound is a decent indicator apparatus for assessing the fetal weight. Expanded placental thickness isn't analytic of a particular problem however may add to the administration of a hatchling in danger.

Eltagy et al. [7] decided predominance of sonographically thick placenta, connection of thick placenta to fetal and maternal intricacies during pregnancy and the result of these pregnancies. They presumed that expanded placental thickness isn't analytic of a particular issue however may add to the administration of an embryo in danger. Estimation of Placental Thickness by U/S is a decent indicator apparatus for assessing the fetal weight. Thick placenta is related with higher rate of low gestational age and low birth weight. Ultrasound frames a promptly accessible, genuinely protected, and shapes a compelling nonobtrusive teqnique. A thick placenta ought to be viewed as a danger factor and needs great development during the remainder of pregnancy.

Azagidi et al. [3] sonographically estimated the PT at the degree of umbilical rope inclusion site. PT has a straight relationship with GA, fetal development boundaries, and EFW and can be utilized alongside other fetal development boundaries to expand the exactness for anticipating GA in typical pregnancies, particularly when the patient isn't sure or doesn't have any acquaintance with her LMP. An anomalous PT for the comparing GA should raise the doubt of basic fetal or maternal illness condition. The nomogram created can be utilized to ascertain the GA with negligible mistake. It is proposed that estimation of PT be completed regularly during obstetric ultrasound examines.

Madazli et al. [13] indicated that placenta from IUGR cases with unusual umbilical supply route doppler velocimetry had an essentially expanded number of villous infarcts, cytotrophoblastic multiplication and thickening of villous trophoblastic cellar film. Unusual placental pathology was essentially connected with anomalous umbilical course doppler velocimetry. The patients with irregular doppler velocimetry had lower mean birth weight.

Kartheek et al. [12] found that perinatal mortality 1 (9.09\%), Apgar Score 10 (90.9\%), IUGR 1 (9.09\%) are higher in cases with irregular doppler stream contrasted with typical cases. Cases with ordinary doppler shows more number of vaginal conveyances $87.18 \%$ though cases with irregular doppler shows more number of cesarean area conveyances $6(54.54 \%)$. They presumed that placental anomalies relate well with the variables causing high danger pregnancies and the ensuing maternal and fetal results. Placental assessments may help in better comprehension of the components of placental dysfunctions.

\section{Conclusion}

So there is measurably critical positive relationship between's placental distance across and EFW, BPD, FL, $\mathrm{AC}$ and umbilical course stream by Doppler. Estimation of placental thickness by U/S is a decent indicator apparatus for assessing the fetal weight.But Increased placental thickness isn't demonstrative of a particular issue however may add to the administration of a hatchling in danger.

\section{References}

[1] A.N.Abdelhamid, T.M.Sayyed, A.H.EShahin, M.A.Zerban. Menoufia Medical J.,, Vol.32, PP.1406-1410, 2019.

[2] E.Aydin, A.N.Bulut. Evaluation of the relationship between placental thickness and obstetric Doppler parameters during the second trimester. Gynecol Obstet Reprod Med, Vol.4, PP.25,2019.

[3] A.S. Azagidi, B.O.Ibitoye, O.N. Makinde, B.M. Idowu, A.S. Aderibigbe. Fetal gestational age determination using ultrasound placental thickness. J Med Ultrasound, Vol.28, , PP.17-23,2020.

[4] H. Azpurua, E.F. Funai, L.M. Coraluzzi. Determination of placental weight using twodimensional sonography andvolumetric mathematic modeling. Am J Perinatol, Vol.27(2), , PP.151-60, 2010.

[5] F.G. Cunningham, K.J. Leveno, S.L. Bloom. Williams's obstetrics, $23^{\text {rd }}$ edition. United States ofAmerica: McGraw-Hill Companies. Chapter 3, Maternal and Fetal Anatomy and Physiology, Vol.5, , PP.54, 2010.

[6] U.Elchalal, Y.Ezra, Y.Levi. Sonographically thick placenta: a marker for increased perinatalrisk: a prospective cross-sectional study. Placenta, Vol.21, , PP.268-72,2000.

[7] A.H. Eltagy, A.O. Abd Elmotaal , I.G.I Alhateem. The prevalence of sonographically thick placenta and its effect on the fetal and maternal outcome. AIMJ, Vol.7,, PP.249-257, 2020.

[8] R.Gagnon. Placental insufficiency and its consequences. Eur J Obstetr Gynecol Reprod Biol, Vol.110, , PP.99-107,2003.

[9] A.H. Hamdy, A.O.Al Saeed, T.M. Mostafa, B.R. Abdel-Aziz. Role of measurement of placental thickness and diameter at the third trimester using two dimensional ultrasound in determination of low birth weight. AIMJ, Vol.9, PP.19-25, 2020.

[10] C.R. Holroyd, N.C. Harvey , S.R. Crozier. Placentalsize at 19 weeks predicts offspring bone mass atbirth: findings from the Southampton Women's Survey. Placenta, Vol.33(8), , PP.623629,2012.

[11] N.W. Jones, N.J. Raine-Fenning, H.A. Mousa, E. Bradley, G.J. Bugg. Evaluating the intra- and inter-observer reliability of three-dimensional ultrasound and power Doppler angiography (3DPDA) for assessment of placental volume and vascularity in the second trimester of pregnancy. 
Ultrasound in Med. and Biol, Vol.37(3),, PP.376-385, 2011.

[12] B.V.S.Kartheek, B.Atla, U.Prasad, U.Namballa, S. Prabhakula. A study of placental morphology and correlation with colour doppler ultrasonography, maternal and neonatal outcome in high risk pregnancies. Int J Res Med Sci, Vol.6, , PP.33649,2018 .

[13] R. Madazli, A. Somunkiran, Z. Calay, S. Ilvan, M.F. Aksu. Histomorphology of the placenta and the placental bed of growth restricted foetuses and correlation with the Doppler velocimetries of the uterine and umbilical arteries. Placenta, Vol.24(5), PP.510-6,2003.

[14] S.Porat, B.Fitzgerald, E. Wright, Placental hyperinflation and the risk of adverse perinatal outcome. Ultrasound Obstet Gynecol, Vol.4,, PP.77$115,2013$.

[15] T.Tongsong, P.Boonanurak. Placental thicknessin the first half of pregnancy:J ClinUltrasound, Vol.9,, PP.32: 231,2004.

[16] J.Zhou, Y.Xiong, Y.Ren, Y.Zhang, X.Li , Y.Yan. Three-dimensional power Doppler ultrasonography indicates that increased placental blood perfusion during the third trimester is associated with the risk of macrosomia at birth. J Clin Ultrasound, Vol.2, PP.1-8,2020.

[17] K.Benirschke, G.Burton. J.Baergeb. Pathology of the human placenta sixth ed.,Springer,Heidelberg, Vol.8, PP.88-112,2012.

[18] R. Giordano, A. Cacciatore, M. Romano, B. Obstetric LaRose, I. Fonti, R. Vigna. Uterine Artery Doppler Flow Studies in obstetric practice J. Prenat.MED, Vol.4,, PP.59-62, 2010.

[19] 1.Preeti BaGhe, 1.Vinita Bahe, S.raShmi Paramhan, V.POmila Sachde, SOnal Onkar.Correlation of Placental Thickness Estimated by - Ultrasonography with Gestational Age and Fetal Outcome .DOI: IJNMR, Vol.13676,, PP.2057,2015.

[20] K. K. SuReSh, R. Ajey BhAgwAt. Ultrasonographic Measurement of Placental Thickness and its Correlation with Femur LengthDOI, Vol.10, PP.25487:2244,2017.

[21] N.Salavati, A.u.sovio, R.plitman mayo, D.S.Charnock-jones, G.C.S.Smith. Placenta (the relationship between human placental morphometry and ultrasonic measurements of utero-placental blood flow and fetal growth, Vol.12,, PP.88-100,2015. 